\title{
Valuation of swing options with supplier flexibility - switching and recall features: a methodology note
}

\author{
S. Persad \\ Hess Corporation, USA
}

\begin{abstract}
This paper presents a method for valuation and risk/sensitivity analysis of swing contracts with switching and/or recall features. Standard swing options allow natural gas customers to exercise a limited number of call features over a period (month) to select the optimal amount of energy to receive each period (day) at a given strike price. More complex versions of these instruments also allow the supplier some flexibility. Two supplier-friendly features are discussed in this paper: (1) Switching features: the supplier must fulfil the buyer's nomination in full, but has a choice of which commodity/delivery location to supply (2) Recall Features: the supplier may recall some of the buyer's nomination amount. This paper offers an algorithm for backward solution. The method draws from Jaillet, Ronn and Tompaidis (2004, Valuation of commodity based swing options. Management Science Vol. 50, Iss. 7, pp. 909-921) by using a forest of trees to represent remaining swing rights, but extends it to a two player (customer and supplier) setting. At each stage the players gather the relevant nodes to which they can transition and engage in a Stackelberg game to determine the optimal action. At each point, the game's outcome is determined employing the game theoretic concept of subgame perfect Nash equilibrium. Because different locations for gas delivery are essentially separate but related markets, the underlying prices to be constructed are two gas locations with a specified correlation. This paper employs Rubenstein's ("Somewhere Over the Rainbow", RISK 4 (November 1991), pp. 63-66; "Return to Oz", RISK 7 (November 1994), pp. 67-71) pyramid methodology for modelling correlated asset price processes. In addition, we adapt the Hull and White (1994, Numerical Procedures for Implementing Models I: Single Factor Models, Journal of Derivatives Vol 2, pp 37-48) procedure for incorporating drift so that our price pyramid captures the mean reversion feature of natural gas. Finally we make adjustments so that the simulation captures seasonality of natural gas prices.

Keywords: path-dependent option pricing, correlated trinomial tree, lattice, swing options, energy options.
\end{abstract}




\section{Introduction}

Commonly used in the natural gas market, swing contracts allow customers some flexibility is choosing the delivery time and amount of their gas. They may exercise a limited number of call features to select the optimal amount of energy to receive each period. For an ordinary swing, the valuation becomes a backward induction problem (with multiple states for number of calls remaining).

More complex versions of these instruments also allow the supplier some flexibility. Two supplier-friendly features are discussed in this paper: Switching features: the supplier must fulfil the buyer's nomination in full, but has a choice of which commodity/delivery location to supply (2) Recall Features: the supplier may recall some of the buyer's nomination amount. The first option allows the supplier to take benefit from location or commodity price differentials. The second feature allows the supplier to act optimally under shortages or upward sloping supply curves.

This paper provides the highlights of the full methodology and empirical analysis of this pricing algorithm as is fully detailed in Persad [5].

\subsection{The specifics of a swing contract}

A vanilla swing contract specifies a strike price (or index), a contract period $\mathrm{T}$, and the length of a subperiod t. The contract specifies that over this contract period (e.g., 30 days), the customer must purchase a given amount $\mathrm{M}$ of gas by taking delivery of up to $\mathrm{m}$ on some customer-selected subperiods (e.g. a day). The number of subperiods on which a customer can choose to take deliver is $\mathrm{T} / \mathrm{t}$. In the contracts the daily maximum, $\mathrm{m}$, is such that $\mathrm{m}^{*} \mathrm{~T} / \mathrm{t}>\mathrm{M}$ and $\mathrm{m}<\mathrm{M}$. That is, in order to reach the contractual quantity $\mathrm{M}$, the customer must exercise on less that the available number of subperiods. In this manner, his/her choice of which day to exercise becomes nontrivial. The customer will have $\mathrm{c}$ swing rights where $\mathrm{c}=\mathrm{M} / \mathrm{m}$.

A swing contract with switching feature grants the supplier a fixed number of switching rights $\mathrm{s}$, where $\mathrm{s}$ is less than or equal to $\mathrm{c}$. That is, the number of switching features is less than the number of swing rights. Each switching right grants the supplier to deliver a substitutable fuel or the same fuel from a different pricing location. For simplicity, we assume that the customer is indifferent between the two fuels. However, the non-trivial Stackelberg game outlined below would easily incorporate a distinction between the two fuels from the customer's point of view.

A swing contract with recall feature allows the supplier to buy back, at a contractually specified price, a certain amount of the daily swing amount if nominated. This is valuable in times of fuel shortages or if the cost of obtaining the supply will increase far beyond the specified strike in the swing contract.

\subsection{Previous work}

Previous work on swing contracts have been examined by Joskow [6], Jalliet et al [1], Barbieri and Garman [7], Pilipovic and Wengler [8], and Harrbrucker and Kuhn [9]. 


\subsection{Overview of methodology}

The method draws from Jaillet et al [1] by using a forest of trees to represent remaining swing rights, but extends it to a two player (customer and supplier) setting. At each stage the players gather the relevant nodes to which they can transition and engage in a Stackelberg game to determine the optimal action. At each point, the game's outcome is determined employing the game theoretic concept of subgame perfect Nash equilibrium. Because different locations for gas delivery are essentially separate but related markets, the underlying prices to be constructed are two gas locations with a specified correlation. This paper employs Rubenstein's [2,3] pyramid methodology for modelling correlated asset price processes. In addition, we adapt the Hull and White [4] procedure for incorporating drift so that our price pyramid captures the mean reversion feature of natural gas. Finally we make adjustments so that the simulation captures seasonality of natural gas prices.

\section{A correlated process for prices}

Because different locations for gas delivery are essentially separate but related markets, the underlying prices to be constructed are two gas locations with a specified correlation. This paper employs Rubenstein's [2, 3] pyramid methodology for modelling correlated asset price processes. In addition, we adapt the Hull and White [4] procedure for incorporating drift so that our price pyramid captures the mean reversion feature of natural gas. Finally we make adjustments so that the simulation captures seasonality of natural gas prices.

We assume that the price processes are Ornstein Uhlenbeck form with correlation rho.

$$
\begin{gathered}
d X_{t}=\alpha_{X}\left(\theta_{X}-X_{t}\right) d t+\sigma_{X} d W_{X} \\
d Y_{t}=\alpha_{Y}\left(\theta_{Y}-Y_{t}\right) d t+\sigma_{Y} d W_{Y} \\
d W_{t} d W_{t}=\rho d t
\end{gathered}
$$

To create a lattice, analogous to Rubenstein's pyramid, but with enough depth to capture mean reversion without collapsing the tree, we settle on using a nine point transition. That is, at time from point $\mathrm{A}$ in time $\mathrm{t}$, the prices can transition to any of the nodes in the diagram below (including staying the same).

The pair at each node indicates the movement of prices $\mathrm{X}$ and $\mathrm{Y}$, where $\mathrm{U}$ indicates up, $\mathrm{M}$ indicates middle (no move) and $\mathrm{D}$ indicates down. The values at each node are given below:

$$
\begin{gathered}
(U, D)=\left\{\Delta X,\left(\rho-\sqrt{1-\rho^{2}}\right) \Delta Y\right\} \\
(U, M)=\{\Delta X, \rho \Delta Y\} \\
(U, U)=\left\{\Delta X,\left(\rho+\sqrt{1-\rho^{2}}\right) \Delta Y\right\} \\
(M, D)=\left\{0,\left(-\sqrt{1-\rho^{2}}\right) \Delta Y\right\}
\end{gathered}
$$




$$
\begin{gathered}
(M, M)=\{0,0\} \\
(M, U)=\left\{0,\left(\sqrt{1-\rho^{2}}\right) \Delta Y\right\} \\
(D, D)=\left\{-\Delta X,-\left(\rho+\sqrt{1-\rho^{2}}\right) \Delta Y\right\} \\
(D, M)=\{-\Delta X,-\rho \Delta Y\} \\
(D, U)=\left\{-\Delta X,-\left(\rho-\sqrt{1-\rho^{2}}\right) \Delta Y\right\}
\end{gathered}
$$

where

$$
\begin{aligned}
& \Delta X=\sigma_{X} \sqrt{3 / 2 \Delta t} \\
& \Delta Y=\sigma_{Y} \sqrt{3 / 2 \Delta t}
\end{aligned}
$$

The shape is a parallelogram rather than a square in order to allow the tree to recombine for the changes in the $\mathrm{Y}$ price while leaving correlations simple to parameterize. The probabilities of moving to each node are denoted by $\mathrm{P}$ (xmove, ymove). We have six natural conditions: two expected values, two means, a correlation and a requirement that the probabilities sum to one. The remaining three conditions are established to ensure that the tree starts off equally likely to move in any direction and to ensure that both prices moving upward is as likely as both prices moving downward and that $\mathrm{X}$ increasing and $\mathrm{Y}$ decreasing is as likely as $\mathrm{Y}$ decreasing and $\mathrm{X}$ decreasing. This can be changed by the user to suit the empirical properties of the underlying prices in question.

Now our price simulation looks like a pyramid with today's change $(0,0)$ at the peak. As we move through time we move toward the base of the pyramid. The only distinction is that the pyramid is somewhat skewed as it is the stack of smaller parallelograms. At each time step, the size of the parallelogram is $(2 t+1)$ $\mathrm{x}(2 \mathrm{t}+1)$ as $\mathrm{dy}$ and $\mathrm{dx}$ can have arrived at, at most, $\mathrm{t}-1$ downward steps or $\mathrm{t}-1$ upward steps until time t-1. Because the tree is recombining, we do not need to store the pyramid, but merely the base. Then we can draw the relevant values at each time step by choosing an increasingly larger dimensioned parallelogram.

The next step is to incorporate mean reversion. We follow the method of Hull and White [4] who construct a mean reverting process in a trinomial tree. They establish three branching patterns and then solve for the probabilities in each. Which branching pattern is relevant is determined by whether or not the price has fallen below or risen above thresholds that depend on the mean.

In our model we must consider nine branching patterns: (1) the standard pattern in figure 1, and the following (2) $\mathrm{X}$ is too high, (3) $\mathrm{X}$ is too low, (4) $\mathrm{Y}$ is too high, (5) $\mathrm{Y}$ is too low, (6) $\mathrm{X}$ is too high and $\mathrm{Y}$ is too low, (7) $\mathrm{X}$ is too high and $\mathrm{Y}$ is too high, (8) $\mathrm{X}$ is too low and $\mathrm{Y}$ is too low, (9) $\mathrm{X}$ is too low and $\mathrm{Y}$ is too high. These branching patterns are illustrated in figure 2. Probabilities are solved for using similar conditions to those required in the ordinary branching pattern. In addition we establish threshold values for $\mathrm{X}$ and $\mathrm{Y}$ so that the probabilities do not become negative. The equations can be solved for the probabilities explicitly (using for example, Matlab's symbolic toolbox) or they 
can be solved numerically. The full results are provided in an the paper Persad [6]. Now our price processes can be envisioned not as a pyramid, but rather as an obelisk, also decreasing storage used.

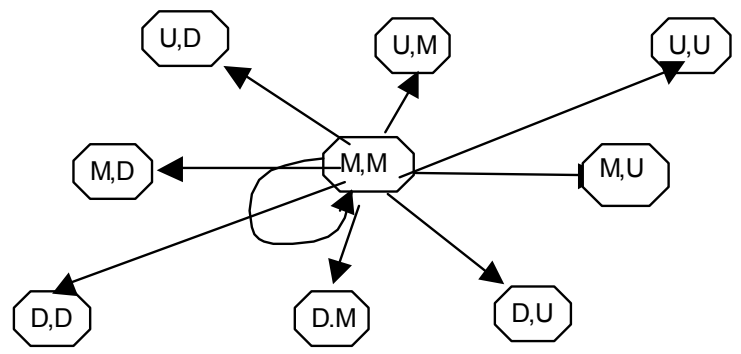

Figure 1: Transition pattern.
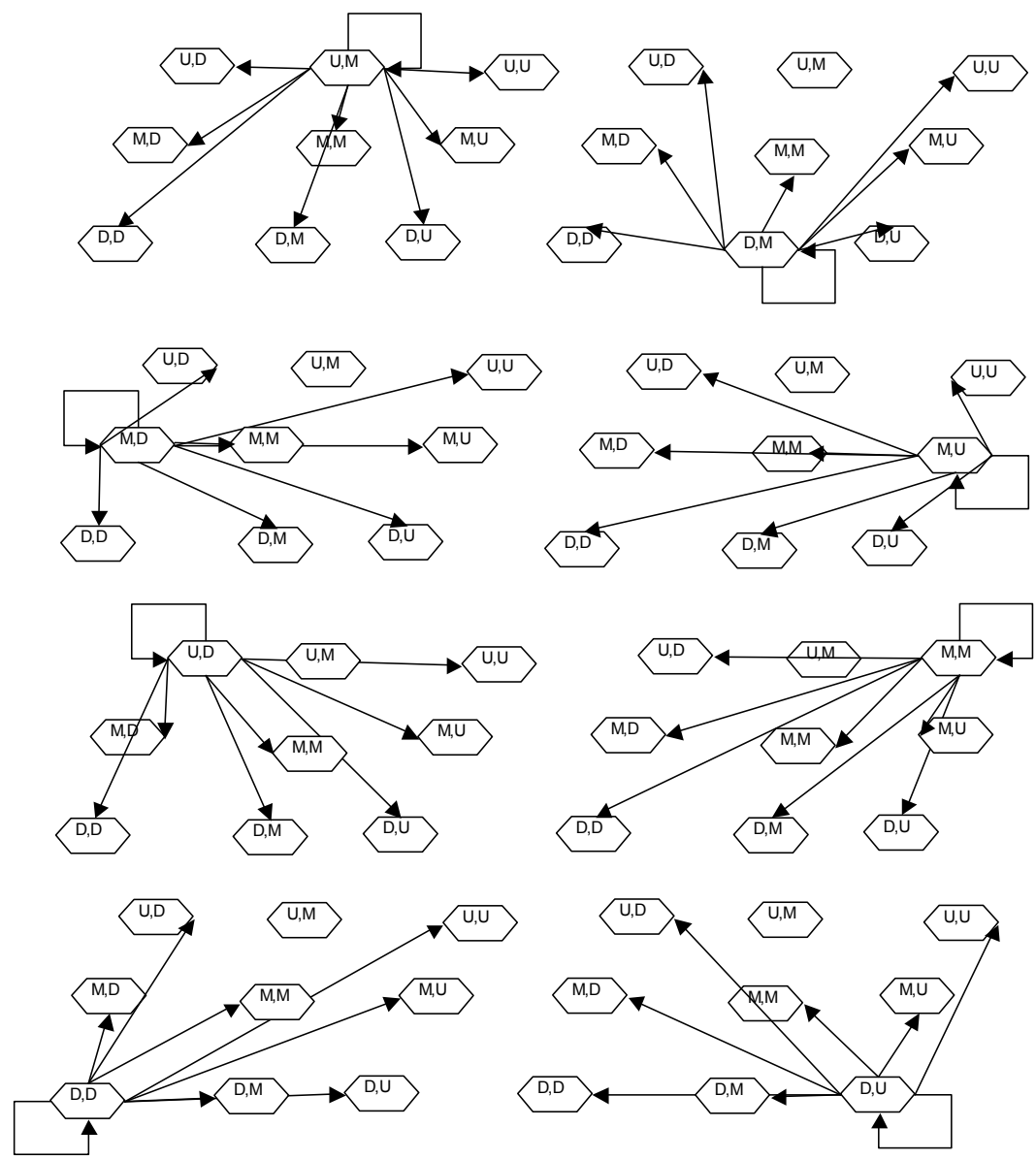

Figure 2: Alternative branching patterns. 
Finally we would like to perturb the tree in order to take into account the current market's expectation of prices in the future. In order to do this we adjust the obelisk at each time step so that in expectation we reach the forward price. This methodology is described in detail in Jaillet, et al [1]. At the end of this step, our price process, can be conceptualised as a crooked obelisk that bends one way or the other in the shape of the forward curve.

In order to assess the reasonableness of our price process, the obelisk is traversed for a large number of paths. The calculated probabilities at each time step, create a probability density function (and therefore a cumulative distribution function). We can make random draws from a uniform distribution to see what our next move should be. Doing this for the entire number of timesteps for a large number of paths will produce several simulations which can then be examined to assess whether they possess the desired behaviour. The results are included in the empirical analysis of this methodology, Persad [5].

\section{Valuation methodology}

The method draws from Jaillet et al [1] by using a forest of trees to represent remaining swing rights, but extends it to a two player (customer and supplier) setting. At each stage the players gather the relevant nodes to which they can transition and engage in a Stackelberg game to determine the optimal action. At each point, the game's outcome is determined employing the game theoretic concept of subgame perfect Nash equilibrium.

For the case of ruthless exercise, the maximum daily amount is transacted for each swing and substitution. Two conditions are sufficient for ruthless exercise (1) if the customer finds the option in the money, he/she will demand the full quantity (demand curve is flat) and (2) in which the supplier can switch fuel but offers any amount up to the maximum daily amount, that the customer demands (supply curve is flat). In the case of ruthless exercise, the equilibrium of the Stackelberg game reduces to the simple expression:

$$
\max \left\{0+U_{c, t+1}\left(E_{s}\right),-\max \left(K-X+U_{s, t+1},\left(E_{c}+1, E_{s}\right), K-Y+U_{s, t+1}\left(E_{c}+1, E_{s}+1\right)\right)\right\}
$$

where Es and Ec denote the number of substitution rights or swing rights used and Us,t and Uc,t denote the utility or payoff to the customer or the supplier at time t.

Some contracts include a recall feature, in which the supplier also has the option to provide less than the maximum daily nomination amount. This arrangement is reasonable when the supplier must deal with shortages or, in the less extreme case, an upward sloping (as opposed to flat) supply curve. Intuitively, the supplier may agree to meet demand to a less than full customer request on a given day if the supply for the first lots fuel comes at a lower unit price than additional lots. This would be the situation if there is a regional shortage or pipeline disruption.

This case allows non-ruthless exercise; the equilibrium may call for transacting less than the maximum daily nomination quantity. The customer and the supplier then each influence the other's decision-making. The customer may 
then want to wait to exercise a swing right when it is more certain of being fulfilled. The supplier may want to save a recall right against future possible calls.

For each decision node, the optimisation problem involves checking three state spaces for the next period: the customer does not call (and the supplier does not use his/her recall or substitution right), the customer calls and the supplier does not substitute, and the customer calls and the supplier substitutes. In this way, the program does not need to hold the entire future state space in memory at one time. Instead it can just call the ones that are necessary.

\section{Results and conclusions}

The pricing results can be compared to a number of limiting cases. First, we know that the swing option with switching grants additional flexibility to the supplier. Since this is a zero-sum game, such flexibility must come at the customer's expense. Therefore the swing option with recall feature must trade at a discount to the standard swing option. The standard swing option may, in turn, be viewed as a basket of American options with the additional constraint that the validity (ability to exercise) the option on any given day depends on the number of options exercised in the past. That is, there is a limited number of swing rights strictly less than the number of possible periods of exercise. Therefore the standard swing option should trade at a (severe) discount to a basket of American options. Because American options on are traded and publicly priced, we are able to compare our results to valid market data. This comparison is reported in the empirical examination of this method as reported in Persad [5].

We can also subject the price methodology to competition. An alternative method of valuation would be to use least-squares Monte Carlo of the type proposed by Longstaff and Schwartz [10]. For practical purposes, this method is not favored because the state space (number of price paths $\mathrm{x}$ number of customer swing rights $\mathrm{x}$ number of supplier recall rights) is large enough to considerably retard computation time. However, it is a valid computational alternative whose merits must also be examined. These comparisons have been performed and are reported in Persad [5].

\section{References}

[1] Jaillet, P., Ronn, E., and Tompaidis, S., 2004, Valuation of commodity based swing options. Management Science Vol. 50, Iss. 7, pp. 909-921.

[2] Rubenstein, M. "Somewhere Over the Rainbow", RISK 4 (November 1991), pp. 63-66.

[3] Rubenstein, M. "Return to Oz", RISK 7 (November 1994), pp. 67-71.

[4] Hull, John C., and Alan White, 1994, Numerical Procedures for Implementing Models I: Single Factor Models, Journal of Derivatives Vol 2, pp 37-48.

[5] Persad, Sandhya, 2008, "Valuation of Swing Options with Supplier Flexibility - Switching and Recall Features: an empirical analysis". 
[6] Joskow, 1987, “Contract Duration and Relationship-Specific Investments: Empirical Evidence from Coal Markets, American Economic Review 77, pp 168-185.

[7] Barbieri, A. and Garman, M., 1997, "Ups and Downs of Swing” Energy and Power Risk Management, 2.

[8] Pilipovic, D and J. Wengler, 1998, "Getting into the Swing," Energy and Power Risk Management, 2.

[9] Haarbrucker, G. and Kuhn, D., 2006, "Valuation of Electricity Swing Options by Multistage Stochastic Programming," Working Paper, Institute for Operations Research and Computational Finance, University of St. Gallen.

[10] FA Longstaff; ES Schwartz, "Valuing American options by simulation: a simple least-squares approach," The Review of Financial Studies; Spring 2001; 14, 1; ABI/INFORM Global pg. 113. 